\title{
Contributorships are not weighable to be equal
}

\author{
Khaled Moustafa*
}

\begin{abstract}
A new trend to assign some authors as 'first coauthors' is noticeable in scientific publications as a statement highlighting that two or more authors have 'contributed equally' to a reported work. However, the requirements of scientific rigor, honesty and accuracy in academic standards make such statements invalid, and thus should be avoided. A potential solution is to specify the role of each coauthor, from study conception to communication of results, and let readers judge the importance of each contribution by themselves. Alternatively, authors should demonstrate how they contribute 'equally' when they are defined as 'equal contributors'.
\end{abstract}

Keywords: authorship; contributorship; authors contributed equally; author contribution; author inflation; coauthorship.

A growing number of scientific practices and unethical conducts compromise the scientific truth in modern scientific knowledge [1]. New and controversial trends in authorship and contributorship activities are increasingly perceptible in scientific publications. For example, authorship inflation -an increase in the number of authors on a single manuscript- is reported in many domains such as medicine [2] ], physics [ㅁ] and social science [4]. The inflation of authorship, however, is deemed unethical [ㄷ] [으 and is often associated with multiple problems [7]. Given the mass production of $\mathrm{PhD}$ trainees [9] and multiple circumstances of ethical ambiguity in academia where the border between legitimate and illegitimate conduct is sometimes blurred [8] , it is increasingly difficult to avoid conflicts of interest and to allocate credit and academic recognition fairly, particularly among large teams [10] and within transdisciplinary collaborations where authorship criteria are heterogeneous [11]. The heterogeneity of collaborating authors may, indeed, result in low robustness of citation analysis models aimed to study the citation impact of coauthors individually [12].

Under an inflated number of authors, the authorial contribution is also compromised. Readers of scientific publications in different fields may notice that two or more authors are sometimes highlighted as 'equal contributors'. The prevalence of such practice is unknown but it has increased significantly in many journals [13]. From an applied and rigorous scientific perspective, however, a statement of 'equal contributions' cannot be a faithful reflection of real and practical 'equal contributions'. Scientific findings, ideas or results (result-to-result) are not 
'weighable' to be 'equal'. Additionally, so far there is no 'specific algorithm' or 'contributorship model' that can compare and quantify the contribution of each coauthor precisely to justify such statements. Rather, the contributorship and the order of authors are often a matter of discussion and agreement, and even so, it is sometimes a source of conflict and complaints [14]. Even if experimental tasks of a given work can be shared out by two or more authors, the different experiments of a work are not 'equal', neither qualitatively nor quantitatively. We cannot measure the 'weight' of an idea, a qualitative result, or an argument made by an author versus another counterpart made by another author so that an 'equal statement' can be objectively justified. An experiment involving DNA cloning, cell culture or DNA extraction cannot be 'equal' to an experiment involving an immunofluorescence assay, western blot or transcriptome analysis, neither methodologically nor temporally. The scientific interpretations and arguments built on such experiments or on others cannot be equal, either.

The lone way that authors could eventually contribute 'equally' to a work is to repeat it literally, but in such cases the repeat will be considered as a 'replication' rather than an 'equal contribution' sensu stricto. Moreover, this is not the sense that authors mean by 'equal contribution' statements. In other words, when authors proclaim 'equal contributions', they do not mean that they have replicated the same experiments verbatim, but rather they should deserve 'equal' academic recognition as first authors. Even if it is fully possible (and highly recommendable) to replicate experiments to confirm obtained results, the different sections of a written manuscript cannot be 'equal'. For instance, the value or 'weight' of an introduction cannot be literally 'equal' to that of a discussion or methods, supposedly these sections contain the same number of words written 'equally' by each contributor.

It is most likely that the goal to appropriate full citation credits and academic recognition is a strong incentive behind such a statement. Otherwise, following the same logic as the proponents of 'equal contributions', coauthors in the middle or at the end of an authorship list can also contribute 'equally' for the remaining tasks shared by the first contributors; why then an 'equal contribution' statement is notified only for first authors but not for middle or last authors who can also contribute 'equally' to other tasks of the reported work?

If contributorships could be measured quantitatively and precisely, then, an 'equal contribution' statement would be acceptable and comprehensible but from a scientific perspective built on rigor and accuracy, a contributorship cannot be 'equal'. It is simply a fallacy caused by a biased academic evaluation system that is based on granting more credit or importance to first (or last) authors. Equal contribution is just a 'ruse' to attain such specific goals in academic affairs. In objective and truthful scientific information, however, the ends (academic recognition or citation credit) should not justify the means (an absurd statement of equal contributions). If 'equal contributions' should be recognized as an acceptable scientific 


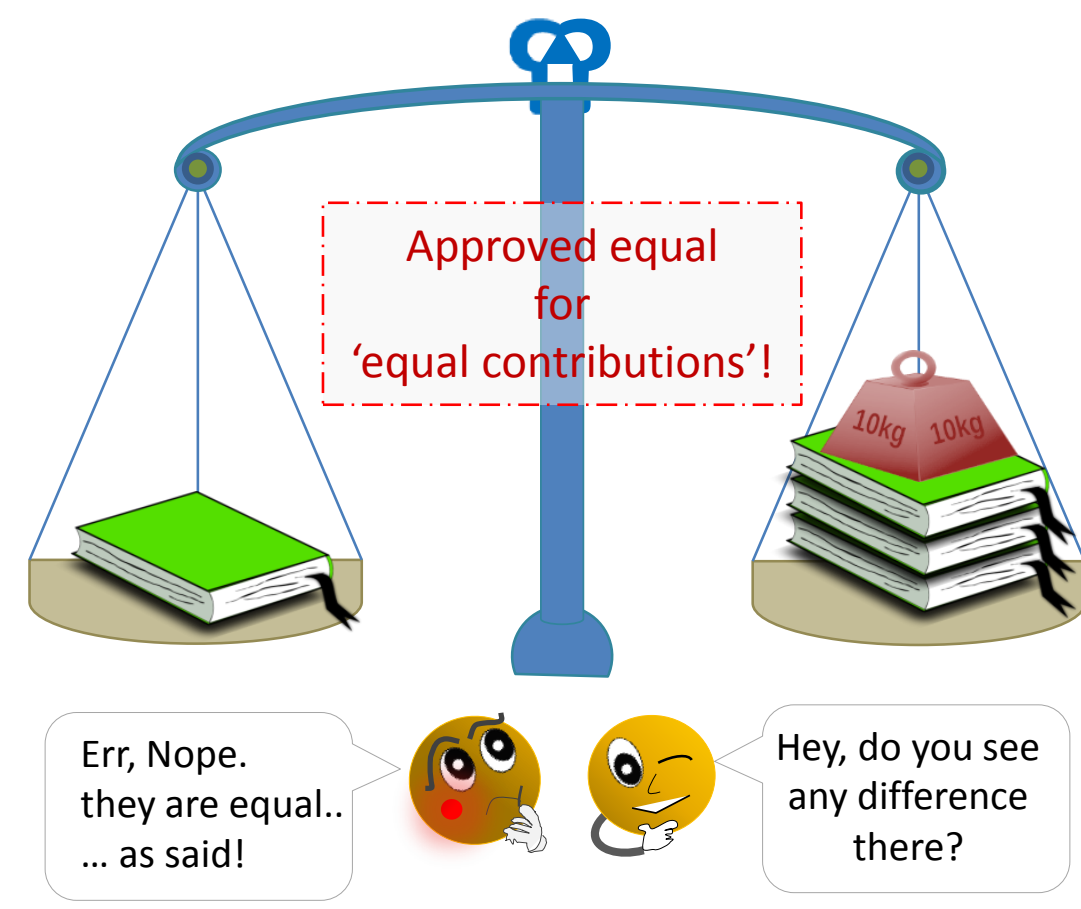

Figure 1. Contributorships cannot be equal. A statement of 'equal contributions' in authorship is an obvious infringe of the accuracy, rigor and consistency of the scientific information. From a rigorous scientific perspective, a statement of 'equal contributions' cannot be a faithful reflection of practical 'equal contributions'. The goal to appropriate full academic credit (promotion, get funding etc.) should not be a proxy for misleading or inaccurate 'equal contribution' statements. Approving such a statement looks like approving that different weights are 'equal'. 
practice, then the corresponding citation credit should also be fractionated equally between 'equal contributors', but not fully appropriated by each of them autonomously. Fractional citation should thus solve some authorial and contributorship issues and make the authorship process more factual than conflictual.

Finally, although satisfying one's ego and looking for the highest credit and largest recognition possible is a human characteristic, honesty and accuracy should be respected alongside the whole scientific enterprise regardless of any credit or recognition sought after. When a practice is common, such as the statement of equal contributions, the commonality of such a practice does not necessarily make it scientifically valid. Authorship is not a 'quantitative measure' properly speaking that can be expressed in equality terms. The question is not whether a first author (as one author) does receive more credit than other coauthors, but in the statement that two or more authors are considered as "first authors" (or "equal contributor") just because the accreditation is given to the authors in the first positions.

Authors should be accurate and rational not only in the way a work is carried out but also in the way the work is written and communicated; authorship contributions cannot be equal and so first co-authorship should be avoided. A potential solution to this issue is to specify the role of each coauthor (who did what) as is already the case in some journals, and to inform research scientists about the irrelevance of such statements, perhaps through targeted educational and mentoring programs intended to helping them ascend their professional careers [15]. Some journals, indeed, require authors to specify the role of each coauthor individually, from the design phase to the communication of the results passing through the experimental work and data analysis. Other journals simply list author names alphabetically, so the problem of order and accreditation is solved, though this would imply 'equal credits' granted to all authors. Whether this is a reflection of real 'equal contributions' is another question, but at least there won't be conflict related to more or less accreditation for only first or last authors. In all cases, the desire to appropriate full publication credit or to get funding or a promotion should not be a proxy for misleading or inaccurate statements, such as the 'equal contribution' of two or more authors.

\section{Disclosure: None.}

\section{References}

1. Treuille, A. and R. Das, Scientific rigor through videogames. Trends in Biochemical Sciences, 2014. 39(11): p. 507-509.

2. Tilak, G., V. Prasad, and A.B. Jena, Authorship Inflation in Medical Publications. Inquiry, 2015. 52.

3. Pritychenko, B., Fractional authorship in nuclear physics. Scientometrics, 2015: p. 1-8. 
4. Henriksen, D., The rise in co-authorship in the social sciences (1980-2013). Scientometrics, 2016: p. 1-22.

5. Shaw, D., Authorship inflation is unethical. EMBO Reports, 2014. 15(11): p. 1106-1106.

6. Bennett, D.M. and D.M. Taylor, Unethical practices in authorship of scientific papers. Emergency Medicine, 2003. 15(3): p. 263-270.

7. Kennedy, D., Multiple authors, multiple problems. Science, 2003. 301(5634): p. 733.

8. Johnson, D.R. and E.H. Ecklund, Ethical Ambiguity in Science. Sci Eng Ethics, 2015.

9. Doran, M.R. and W.B. Lott, $A$ duty of care. Trends in Biochemical Sciences, 2013. 38(1): p. 1-2.

10. Petersen, A.M., I. Pavlidis, and I. Semendeferi, A quantitative perspective on ethics in large team science. Sci Eng Ethics, 2014. 20(4): p. 923-45.

11. Teixeira da Silva, J.A. and J. Dobranszki, Multiple Authorship in Scientific Manuscripts: Ethical Challenges, Ghost and Guest/Gift Authorship, and the Cultural/Disciplinary Perspective. Sci Eng Ethics, 2015.

12. Glänzel, W., S. Heeffer, and B. Thijs, A triangular model for publication and citation statistics of individual authors. Scientometrics, 2016: p. 1-16.

13. Akhabue, E. and E. Lautenbach, "Equal" contributions and credit: an emerging trend in the characterization of authorship. Ann Epidemiol, 2010. 20(11): p. 868-71.

14. Gaeta, T.J., Authorship: "Law" and Order. Academic Emergency Medicine, 1999. 6(4): p. 297301.

15. King, L., Helping early career research scientists ascend the professional ladder. Trends in Biochemical Sciences, 2013. 38(8): p. 373-5.

${ }^{*}$ Email: khaled.moustafa@gmail.com 